\title{
Monopolar high-frequency language mapping: can it help in the surgical management of gliomas? A comparative clinical study
}

\author{
Marco Riva, MD, ${ }^{1}$ Enrica Fava, MD, ${ }^{1,2}$ Marcello Gallucci, PhD, ${ }^{3}$ Alessandro Comi, PhD, ${ }^{2}$ \\ Alessandra Casarotti, PhD, ${ }^{2}$ Tommaso Alfiero, MD, ${ }^{1}$ Fabio A. Raneri, MD, ${ }^{1}$ Federico Pessina, MD, ${ }^{2}$ \\ and Lorenzo Bello, MD ${ }^{1,2}$
}

${ }^{1}$ Neurosurgery, Department of Medical Biotechnology and Translational Medicine, Università degli Studi di Milano, Rozzano (MI), 2Unit of Neurosurgical Oncology, Humanitas Research Hospital, Rozzano (MI), and ${ }^{3}$ Faculty of Psychology, Università di Milano Bicocca, Milan, Italy

OBJECTIVE Intraoperative language mapping is traditionally performed with low-frequency bipolar stimulation (LFBS). High-frequency train-of-five stimulation delivered by a monopolar probe (HFMS) is an alternative technique for motor mapping, with a lower reported seizure incidence. The application of HFMS in language mapping is still limited. Authors of this study assessed the efficacy and safety of HFMS for language mapping during awake surgery, exploring its clinical impact compared with that of LFBS.

METHODS Fifty-nine patients underwent awake surgery with neuropsychological testing, and LFBS and HFMS were compared. Frequency, type, and site of evoked interference were recorded. Language was scored preoperatively and 1 week and 3 months after surgery. Extent of resection was calculated as well.

RESULTS High-frequency monopolar stimulation induced a language disturbance when the repetition rate was set at 3 $\mathrm{Hz}$. Interference with counting $(p=0.17)$ and naming $(p=0.228)$ did not vary between HFMS and LFBS. These results held true when preoperative tumor volume, lesion site, histology, and recurrent surgery were considered.

Intraoperative responses (1603) in all patients were compared. The error rate for both modalities differed from baseline values $(p<0.001)$ but not with one another $(p=0.06)$. Low-frequency bipolar stimulation sensitivity $(0.458)$ and precision (0.665) were slightly higher than the HFMS counterparts $(0.367$ and 0.582 , respectively). The error rate across the 3 types of language errors (articulatory, anomia, paraphasia) did not differ between the 2 stimulation methods $(p=0.279)$.

CONCLUSIONS With proper setting adjustments, HFMS is a safe and effective technique for language mapping. http://thejns.org/doi/abs/10.3171/2015.4.JNS14333

KEY WORDS language mapping; direct electrical stimulation; glioma; awake brain surgery; diagnostic and operative techniques

$\mathrm{T}$ HE current strategy for addressing intrinsic brain lesions, such as gliomas, is aimed at maximal tumor resection while preserving the patient's functional integrity. ${ }^{9,31,33,36-38}$ Achieving these aims can be hampered by the infiltrative nature of gliomas, which can involve essential functional structures. ${ }^{16}$

Intraoperative direct electrical stimulation (DES) coupled with neuropsychological testing has been increasingly recognized as an efficient strategy to improve the ex- tent of resection (EOR) and reduce postoperative morbidity. ${ }^{10,13,18}$ In fact, DES allows identification and localization (mapping) of eloquent structures, at both the cortical and subcortical level. ${ }^{5,15,17-19,30,34,39}$ In addition, a tailored neuropsychological evaluation provides an appropriate cognitive picture of the patient, leading to an objective definition of their cognitive status and guiding resection during language and cognitive mapping. ${ }^{27}$

Language mapping is traditionally performed with

ABBREVIATIONS $A D$ = afterdischarge; $D E S$ = direct electrical stimulation; $E C O G$ = electrocorticography; $E E G$ = electroencephalography; EMG = electromyography; EOR = extent of resection; HFMS = high-frequency monopolar stimulation; HGG = high-grade glioma; LFBS = low-frequency bipolar stimulation; LGG = low-grade glioma; MEP = motor evoked potential.

SUBMITTED February 19, 2014. ACCEPTED April 2, 2015.

INCLUDE WHEN CITING Published online September 25, 2015; DOI: 10.3171/2015.4.JNS14333. 
DES delivered in trains of biphasic pulses at $50-60 \mathrm{~Hz}$ (low frequency) ${ }^{6,7,25,29}$ through a bipolar probe. During testing, DES is applied at both the cortical and subcortical level with the patient awake, allowing identification of the essential sites involved in the various components of language function, schematically classified as articulatory, semantic, phonemic, and syntactic. $5,6,17-19,25,29,30,34$ The technique is feasible and robust, and when it is toggled with tumor resection according to functional boundaries, surgeons can optimize the EOR and simultaneously maintain a high level of the patient's functional integrity. $6,10,13,17-19,34$ The incidence of seizures during awake mapping is usually low. ${ }^{6,13,19}$ However, in patients with a long history of seizures and poor seizure control, ${ }^{7,26}$ low-frequency bipolar stimulation (LFBS) can be associated with a higher risk of seizures on stimulation, even when the modality is used at the lowest useful current intensity. ${ }^{7,40,42}$ The likelihood of performing effective functional mapping can thus be hampered.

High-frequency $(250-500 \mathrm{~Hz})$ multipulse stimulation, ${ }^{41}$ usually consisting of 5 pulses, that is, the train-offive technique, delivered through a monopolar probe was introduced and proved to be effective in mapping motor structures. In fact, high-frequency monopolar stimulation (HFMS) was more effective and less epileptogenic than LFBS during motor mapping. $7,23,35,40,42$

To the best of our knowledge, a systematic analysis of the effect of HFMS for intraoperative language mapping has not been conducted, and thus its use has been limited. ${ }^{3,23}$ In the current study, we explored the use of train-offive HFMS in performing language mapping: the efficacy and safety of this stimulation paradigm were compared with those of the LFBS modality during awake neurosurgical procedures needing language mapping. The impact on neurosurgical performance, in terms of EOR and language outcome, was also investigated.

\section{Methods \\ Patients}

Fifty-nine consecutive patients (Table 1) affected by a presumed glioma were consecutively recruited. Inclusion criteria were as follows: 1) surgical removal of a presumed glioma in the dominant hemisphere performed with asleep-awake anesthesia; 2) lesion site including cortical and subcortical structures relevant for language; 3) standardized neuropsychological evaluation: $;{ }^{27}$ and 4) volumetric MRI studies. All patients gave written informed consent for the surgical and mapping procedures. The local ethics committee approved the study.

\section{Neuroradiological Protocol}

Magnetic resonance imaging was performed preoperatively and postoperatively on a 3-T scanner (Verio, Siemens). Image acquisition and postprocessing were performed as previously described. ${ }^{6}$ Lesion volumes were computed on FLAIR volumetric sequences using manual segmentation with region of interest analysis using the iPlan Cranial 3.0 software suite (Brainlab). FLAIR hyperintense and gadolinium-enhanced signal abnormalities were included in the lesion load for low-grade gliomas
TABLE 1. Demographic and clinical features in 59 patients with glioma*

\begin{tabular}{|c|c|c|}
\hline \multirow[b]{2}{*}{ Parameter } & \multicolumn{2}{|c|}{ No. } \\
\hline & Group 1 & Group 2 \\
\hline No. of patients & 9 & 50 \\
\hline \multicolumn{3}{|l|}{ Sex } \\
\hline Male & 5 & 31 \\
\hline Female & 4 & 19 \\
\hline Mean age in yrs & $38.5 \pm 11.3$ & $43.1 \pm 13.6$ \\
\hline Hand dominance (rt/lt) & $9 / 0$ & $48 / 2$ \\
\hline Median yrs of education (range) & $12(8-17)$ & $11(6-17)$ \\
\hline Seizure at presentation (\%) & $8(88.9)$ & $48(96.0)$ \\
\hline Seizure history >6 mos (\%) & $8(88.9)$ & $21(42.0)$ \\
\hline \multicolumn{3}{|l|}{ No. of AEDs (\%) } \\
\hline 1 & $1(11.1)$ & $24(48.0)$ \\
\hline 2 & $7(77.8)$ & $22(44.0)$ \\
\hline 3 & $1(11.1)$ & $4(8.0)$ \\
\hline $\begin{array}{l}\text { Median KPS score at presentation } \\
\text { (range) }\end{array}$ & $100(90-100)$ & $100(71-100)$ \\
\hline \multicolumn{3}{|l|}{ Main lesion site (\%) } \\
\hline Frontal & $8(88.9)$ & $26(52.0)$ \\
\hline Temporal & $1(11.1)$ & $15(30.0)$ \\
\hline Parietal & 0 & $5(10)$ \\
\hline Insular & 0 & $3(6.0)$ \\
\hline Occipital & 0 & $1(2)$ \\
\hline Insular involvement (\%) & $3(33.3)$ & $10(20)$ \\
\hline \multicolumn{3}{|l|}{ Mean lesion vol in $\mathrm{cm}^{3}$} \\
\hline LGGs (FLAIR) & $39.9 \pm 31.5$ & $28.2 \pm 26.4$ \\
\hline HGGs (T1WI+Gd) & $25.6 \pm 18.6$ & $28.6 \pm 25.8$ \\
\hline \multicolumn{3}{|l|}{ Histology (\%) } \\
\hline \multicolumn{3}{|l|}{ WHO Grade I } \\
\hline Ganglioglioma & & $1(2.0)$ \\
\hline WHO Grade II & $7(77.8)$ & $17(34.0)$ \\
\hline Astrocytoma & 1 & 1 \\
\hline Oligodendroglioma & 4 & 10 \\
\hline Oligoastrocytoma & 2 & 6 \\
\hline WHO Grade III & $1(11.1)$ & $13(26.0)$ \\
\hline Anaplastic astrocytoma & 1 & 4 \\
\hline Oligodendroglioma & & 3 \\
\hline Oligoastrocytoma & 1 & 5 \\
\hline Ependymoma & & 1 \\
\hline WHO grade IV & $1(11.1)$ & $17(34.0)$ \\
\hline Glioblastoma multiforme & 1 & 17 \\
\hline Others (metastases) & & $2(4.0)$ \\
\hline First surgery (\%) & $4(44.4)$ & $27(54.0)$ \\
\hline Median EOR in \% (range) & $100(72-100)$ & $100(53.6-100)$ \\
\hline
\end{tabular}

$\mathrm{AED}=$ antiepilepsy drug; KPS = Karnofsky Performance Scale; $T 1 \mathrm{WI}+\mathrm{GD}=$

T1-weighted imaging with gadolinium enhancement.

* Mean values are expressed with the standard deviation.

(LGGs) and high-grade gliomas (HGGs), respectively, and then were reported in cubic centimeters. Extent of resection was measured on pre- and postoperative MRI studies 
obtained within 48 hours of the end of surgery and was classified as previously reported ${ }^{36}(\mathrm{EOR}=[$ \{preoperative volume - postoperative volume $\}$ /preoperative volume] $\times$ 100).

\section{Neuropsychological Evaluation}

A neuropsychological battery including verbal and nonverbal function assessments was performed. ${ }^{27,29} \mathrm{~Pa}$ tients were evaluated at 3 time points: within 1 week before the surgical procedure (baseline T0) and 1 week (T1) and 3 months (T2) after surgery. Two board-certified neuropsychologists systematically investigated different domains of language. This evaluation was used to depict the wide spectrum of the overall language performance of the patient pre- and postoperatively. Explored domains were as follows: 1) auditory comprehension, 2) words and sentences comprehension, 3) noun and verb naming, 4) phonemic fluency, and 5) semantic fluency.

During the intraoperative session, blocks of 80 items were shown on a laptop screen. Electrical stimulation was applied just before the slide appeared, with the patient unaware of the onset of the stimulation; 2 slides without stimulation were presented after a given stimulation had been applied. Stimulation sites were selected randomly. Stimulation was repeated 3 times at the same site in case of an evoked disturbance, to acknowledge the relationship between DES and an induced response as bona fide.

Intraoperative language disturbances were classified into 3 groups: 1) articulatory (motor) disturbances (anarthria, dysarthria), 2) anomia (latency in response, perseveration, anomia), and 3) paraphasia (semantic paraphasia, phonemic paraphasia, verbal paraphasia, neologisms).

\section{Anesthesia}

Total intravenous anesthesia with propofol and remifentanil was induced, and no muscle relaxants were employed during surgery. We endeavored to prevent intraoperative seizures by closely monitoring electrocorticography (ECoG) and continuous electromyography (EMG) activity. Stimulation was stopped and cold irrigation was applied at the first ictal sign to prevent the build up of a seizure. Whenever the seizure did not stop in a few seconds and the convulsive activity spread to the entire hemibody, a propofol bolus infusion was delivered. Craniotomy and dural opening in the study group were performed with the patients under asleep anesthesia. Mapping procedures were performed after intraoperatively awakening the patient.

\section{Surgical Protocol}

Surgery was performed with the aid of intraoperative cortical and subcortical mapping of motor and language functions, associated with monitoring procedures, as previously described. ${ }^{4,6,7,29}$ Histology was classified according to the (2002) WHO brain tumor classification. ${ }^{22}$

\section{Neurophysiological Monitoring and Mapping Protocol}

A multimodal electrophysiological monitoring procedure was used throughout the surgery, as previously reported. ${ }^{7}$ Cortical and subcortical mapping was performed using an Osiris stimulator (Inomed) by adopting 2 dis- tinct stimulation paradigms: 1) LFBS: $60-\mathrm{Hz}$ biphasic pulses, total duration $1 \mathrm{msec}$, current intensity referring to each phase, interstimulus interval $16.66 \mathrm{msec}$, trains lasting 1-4 seconds; and 2) HFMS: train-of-five monophasic pulses, pulse duration $0.5 \mathrm{msec}$, interstimulus interval 2-4 msec (2 msec for language mapping, 2-4 msec for motor mapping), anodal and cathodal stimulation for cortical and subcortical mapping, respectively, train repetition rate 1-3 Hz. Stimulation was applied for language and motor mapping. Data regarding language mapping exclusively are reported. Neurophysiological recordings of brain and muscle activity were acquired using a poligraphic electroencephalography (EEG) system (Grass Technologies) and multimodal equipment (ISIS IOM system, Inomed). ${ }^{7}$ Subcortical mapping was alternated with resection, using the same current threshold applied for cortical mapping.

Language mapping was performed first by stimulating the ventral premotor cortex in an attempt to stop counting (speech arrest, or anarthria) to identify the intensity of the working current, defined as the minimal intensity that produced anarthria without inducing any type of epileptiform activity. The working current was assessed for both types of stimulation, that is, LFBS and HFMS. During mapping, this current intensity was checked by repeatedly stimulating the ventral premotor cortex to induce anarthria, and the intensity was eventually adjusted. The same intensity was employed for the noun-naming task and further language evaluation once the working current was established. Ictal events were recorded using ECoG (afterdischarges [ADs] and electrical seizures) and EMG (convulsive seizures) as well as clinical examination.

\section{Statistical Analysis}

Features of the patients are expressed as the mean \pm standard deviation or as the median and range for continuous and categorical variables, respectively.

High-frequency monopolar stimulation was employed with 2 distinct train repetition rates at the beginning of mapping, that is, 1 and $3 \mathrm{~Hz}$; therefore, 2 groups of patients were identified (Group 1 and Group 2, respectively). Low-frequency bipolar stimulation was used in all patients and was compared with the HFMS modality within each group.

Two sets of analyses were applied in Group 2: patientlevel and stimulation-level analyses. The percentage of language errors determined by each type of stimulation in every patient represented the patient-level analyses, whereas the stimulation-level analysis consisted of the evaluation of each stimulation trial in terms of the type of error it had produced. As previously stated, 3 types of evoked language disturbances were considered, and no stimulation data were used as a base rate. The pairwise comparison between the 2 stimulation modalities was completely available in 30 patients in Group 2. In fact, the remaining 20 patients in Group 2 displayed a high risk of intraoperative seizures triggered by LFBS, as demonstrated for motor mapping. ${ }^{7}$ Low-frequency bipolar stimulation was ineffective for language mapping in a high proportion of these 20 patients: no language interference was found even at a high current intensity (up to $12 \mathrm{~mA}$ ). A pairwise 
comparison with HFMS could not be performed, and thus a distinct set of analyses was applied.

A random-intercepts binomial model was employed at the patient-level to investigate the differences in counting and naming interference between HFMS and LFBS. The results of these analyses were checked for the following variables: lesion site, tumor volume, histology, first surgery, and log-transformed motor evoked potential (MEP) threshold. A repeated measures ANOVA with Satterthwaite correction was used to compare the current intensity of the 2 stimulation modalities.

A random-intercepts generalized linear model implemented as a series of logistic regressions ${ }^{1,14}$ was used to perform the stimulation-level analyses, using the stimulation outcome as a binary dependent variable. Each data input was associated with 1 of the 3 following conditions: 1) no stimulus, 2) HFMS, or 3) LFBS. Responses were classified as 1) correct, 2) articulatory disturbance, 3) anomia, and 4) paraphasia, as stated above.

The performance of neuropsychological tests at the 3 time points was analyzed using a random-intercepts ANOVA with Satterthwaite correction to model the repeated-measures design of the variables.
Statistical analyses were performed with $\mathrm{R}$ software (http://www.R-project.org), and a p value $<0.05$ was set as significant.

\section{Results}

\section{Initial Evaluation of the Effect of HFMS}

The effect of HFMS on language mapping was initially evaluated in 9 patients with nonenhancing tumors affecting the dorsal portion of the premotor cortex and with poorly defined lesion borders (Group 1; Table 1). Initial stimulation of the ventral premotor cortex with LFBS at 3-4 $\mathrm{mA}$ induced seizures in 2 patients and ADs and sporadic partial seizures in the following 2 patients (Fig. 1).

High-frequency monopolar stimulation was then employed in these 4 patients, starting with the stimulation parameters reported in the literature during motor mapping, that is, with a repetition rate of $1 \mathrm{~Hz}$. The HFMS (10-18 $\mathrm{mA}, 1 \mathrm{~Hz}$ ) induced an arrest in counting in all patients, without the occurrence of epileptic phenomena. Identical HFMS (10-18 mA, 1 Hz) was used at the subcortical level, where it induced both face muscle recruitment and anarthria, allowing identification of the resection margins
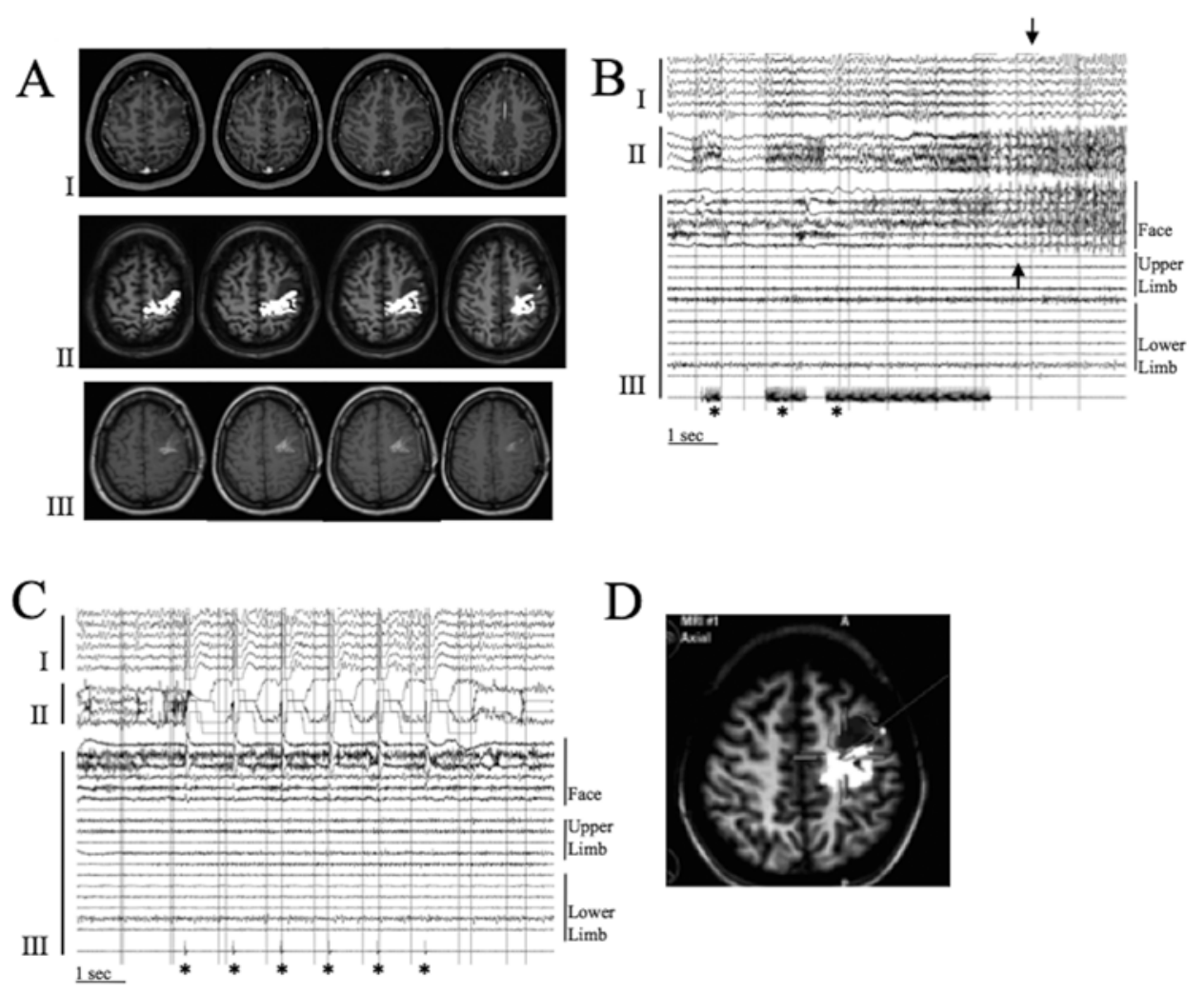

FIG. 1. Group 1. A case of LGG involving the dominant dorsal premotor cortex area. A close relationship with the corticospinal tract (All) is present. The patient had a long history of partial seizures (speech arrest) poorly controlled by antiepilepsy drugs. Language mapping was started with LFBS (3 mA) of the premotor cortex, looking for anarthria and speech arrest (B). The onset of ADs (upper arrow, B) was accompanied by the occurrence of a partial seizure (lower arrow) involving the face muscles, preventing reliable mapping. The mapping was resumed using HFMS $(\mathrm{C})$ at $1 \mathrm{~Hz}$, which allowed us to establish the working current and to complete the cortical mapping. The HFMS at $1 \mathrm{~Hz}$ was also used to perform subcortical mapping and to completely remove the lesion (AIII). Intraoperative screenshot (D) of the subcortical site where HFMS generated anarthria. Al: Preoperative gadoliniumenhanced T1-weighted images. All: Fiber tract reconstructions of the corticospinal tract (white) superimposed on T1-weighted images. Alll: Postoperative gadolinium-enhanced T1-weighted images. BI: ECoG tracings. BII: EEG tracings. BIII: EMG tracings. Cl: ECOG tracings. Cll: EEG tracings. CIII: EMG tracings for muscles belonging to the face, upper limb, and lower limb. Asterisks indicate a stimulus artifact. 
(Fig. 1C and D). No naming tasks were performed with the HFMS. The EOR was $100 \%$ in these first 4 patients.

In the other 5 patients harboring a tumor involving the dorsal portion of the premotor cortex and the upper insula, LFBS (2.5-5 mA) induced ADs in 2 patients on stimulating the ventral premotor cortex; therefore, HFMS (8-10 $\mathrm{mA}, 1 \mathrm{~Hz}$ ) was used. The HFMS effectively induced anarthria without seizure activity. No paraphasias or anomias were observed in these 5 patients during the naming task when HFMS with a repetition rate of $1 \mathrm{~Hz}$ was applied to other sites of the frontal and temporal cortex. An increase in the train repetition rate from 1 to $3 \mathrm{~Hz}$ and the same current intensity (8-10 $\mathrm{mA})$ led to the identification of cortical sites determining paraphasias and anomias. Electromyography detected no evoked muscle potentials during these language disturbances. This adjustment of the stimulation frequency enabled the identification of essential cortical language sites over the frontal cortex as well as the surgical point of entry. When applied with a repetition rate of 3 $\mathrm{Hz}$ at the subcortical level, the HFMS induced phonemic and semantic paraphasias and thus identified as functional boundaries, respectively, the dorsal language tracts (that is, the arcuate and superior longitudinal fascicles) and the inferior frontooccipital fascicle, with no MEPs in these sites (Fig. 2). The EOR was $100 \%$ in 3 patients and $91.4 \%$ and $83.9 \%$ in the remaining 2 . Thus, $3 \mathrm{~Hz}$ was effective as
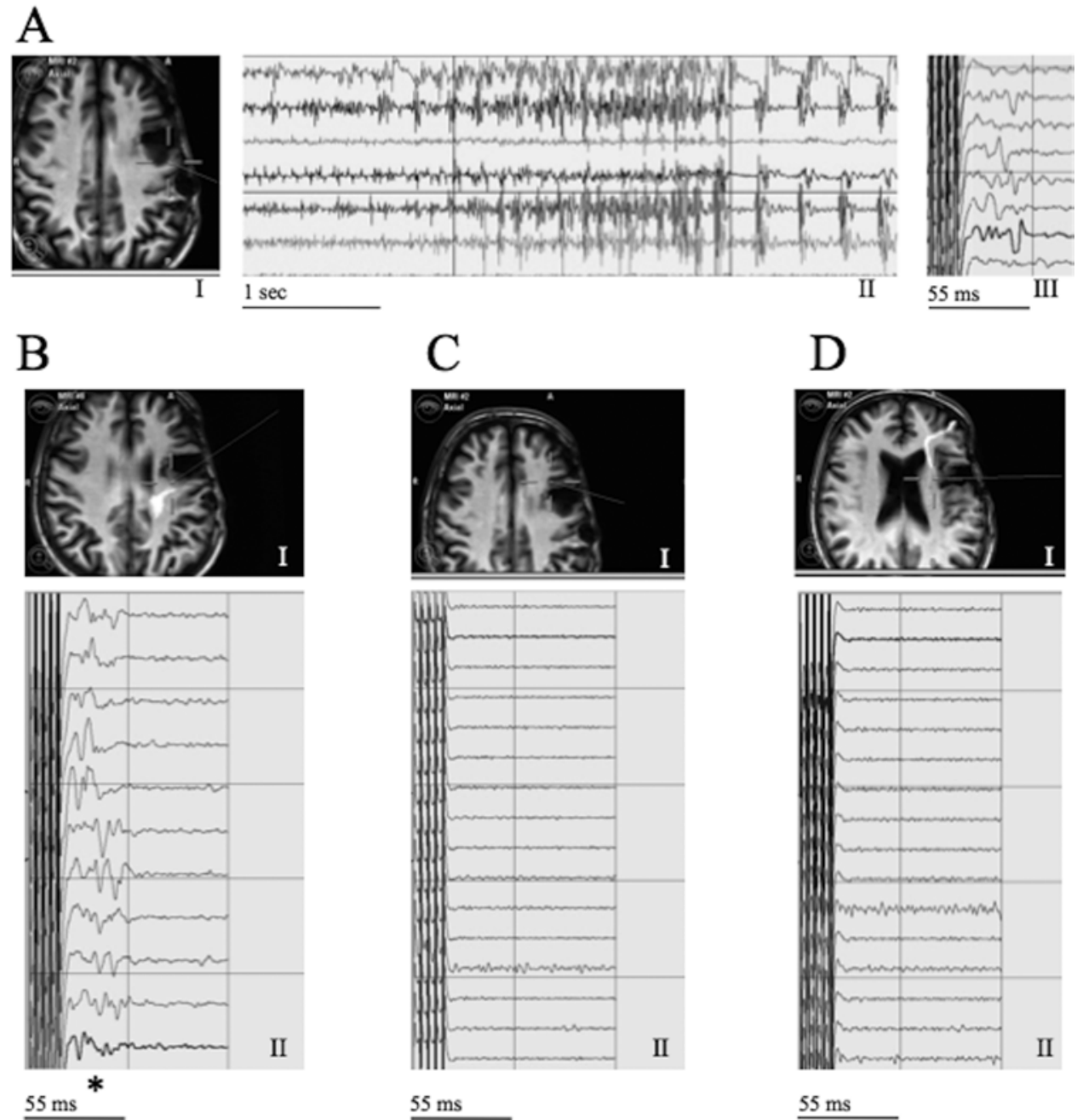

$55 \mathrm{~ms}$

$55 \mathrm{~ms}$

FIG. 2. Group 1. A case of LGG involving the dominant dorsal premotor cortex and the upper insula. Language mapping started with LFBS $(2.5 \mathrm{~mA})$ of the ventral premotor cortex (intraoperative screenshot, Al). The LFBS induced a partial seizure involving face and neck muscles, preventing reliable mapping (All). Mapping was performed with HFMS (1 Hz, AllI), which induced anarthria without $A D s$ or seizures. The repetition rate was then increased to $3 \mathrm{~Hz}$, which generated all types of the language disturbances considered herein over the frontal cortex. The same current intensity $(8 \mathrm{~mA}, 3 \mathrm{~Hz})$ was used to perform subcortical mapping (B-D) and to identify the functional boundaries. It is noteworthy that only stimulation of both the ventral premotor cortex (AIII) and the fibers (BII) generated a motor articulatory disturbance, that is, anarthria (asterisk), along with MEPs in the perioral muscles. Only the mylohyoid muscle MEP is shown. Al: TI-weighted MR image with intraoperative acquisition of the point of interest. All: Continuous EMG during LFBS with recording of the ictal activation of the perioral muscles. Alll: Recording from the mylohyoid muscle upon HFMS ( $5 \mathrm{~mA}, 1 \mathrm{~Hz})$. BI, Cl, and DI: Intraoperative screenshots of the site of induced anarthria and phonemic and semantic paraphasias, respectively. BII, CII, and DII: Corresponding mylohyoid muscle motor responses triggered by stimulation of the premotor component of the corticospinal tract (MEP is present with visible stimulus artifacts) and of the arcuate and inferior frontooccipital fascicles, where no MEPs are present and only the stimulus artifact is visible. 
the repetition rate for language mapping, and it was then applied as such to the subsequent surgeries.

\section{Comparative Study}

A systematic evaluation of the effectiveness of HFMS at $3 \mathrm{~Hz}$ was performed. Pairwise data regarding the 2 distinct stimulation modalities were available in 50 consecutive patients (Group 2) affected by gliomas involving language-related structures.

\section{Patient-Level Analysis}

Counting interference did not differ statistically significantly between patients undergoing LFBS and HFMS $(\chi=1.879, \mathrm{p}=0.17$; Fig. 3 upper I-II and lower I-II). Differences between the 2 modalities were still not statistically significant after controlling for preoperative tumor volume, $(\chi=1.878, \mathrm{p}=0.171)$, lesion site $(\chi=1.547, \mathrm{p}=$ $0.461)$, histology $(\chi=1.621, \mathrm{p}=0.203)$, and recurrent surgery $(\chi=1.459, \mathrm{p}=0.227)$.

A similar performance was observed in the noun-naming task (Fig. 3 upper III-IV and lower III-IV). In particular, LFBS and HFMS induced a naming interference in $24(48 \%)$ and 30 patients $(60 \%)$, respectively. Those percentages were not statistically different $(\chi=1.453, \mathrm{p}=$ 0.228 ). The absence of a statistically significant difference remained after controlling for preoperative tumor volume
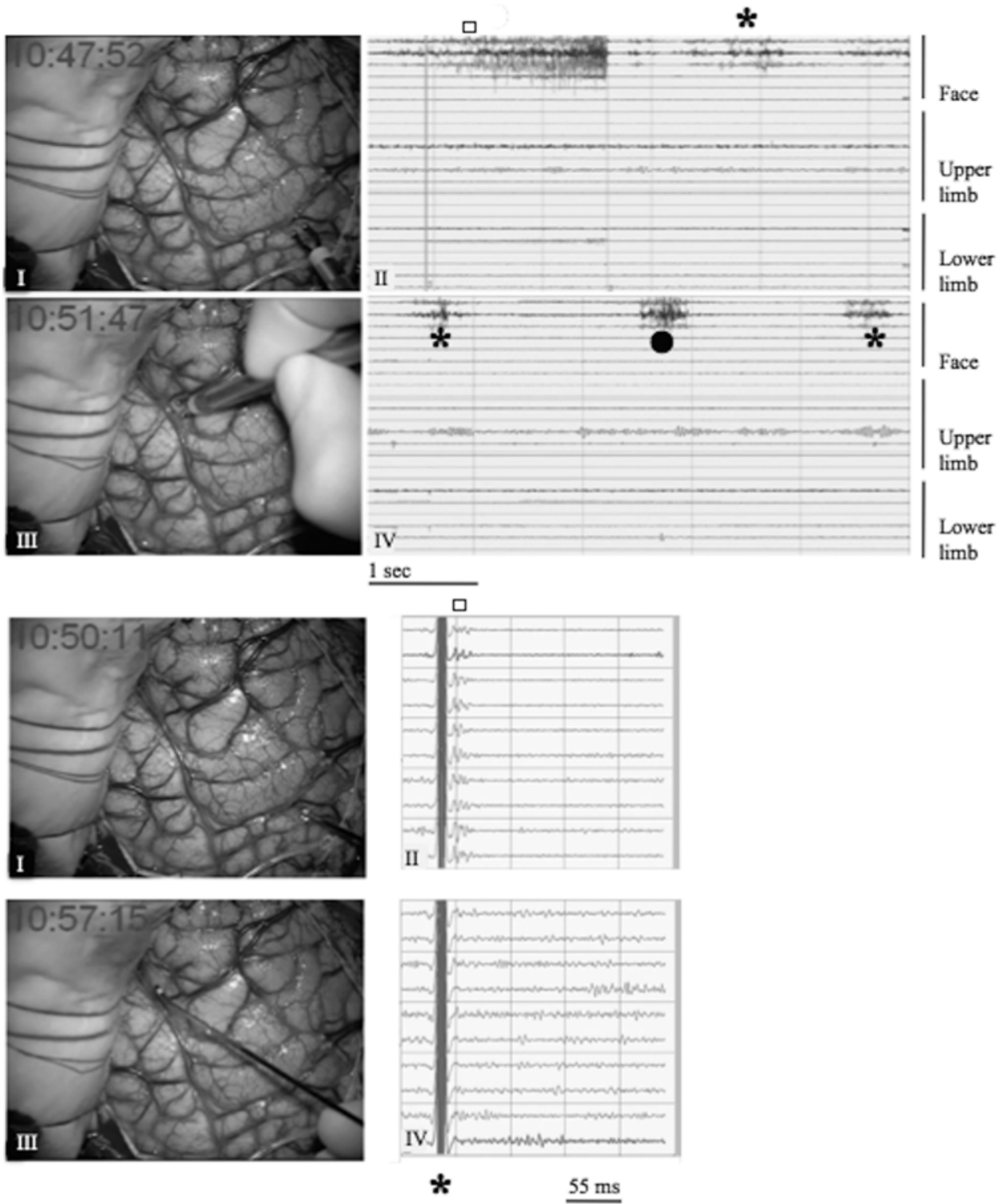

FIG. 3. Group 2. Cortical mapping with LFBS (upper) and HFMS (lower) in a case of dominant frontal LGG. Intraoperative photograph (upper I) depicting LFBS applied (4 mA) to the ventral premotor cortex, generating anarthria (upper II, white box) and thus interfering with normal speech (asterisk). Intraoperative photograph (upper III) depicting LFBS applied (4 mA) to the pars opercularis of the inferior frontal gyrus, inducing anomia (upper IV, black circle). Correct responses also appear (asterisks). Similarly, HFMS (lower I) applied (7 mA, $3 \mathrm{~Hz}$ ) to the same site of the ventral premotor cortex induced anarthria and MEPs (lower II, EMG recording of the mylohyoid muscle). Moreover, HFMS (lower III) applied (7 mA, $3 \mathrm{~Hz}$ ) to the operculum of the inferior frontal gyrus induced a language disturbance without articulatory interference (lower IV, EMG showing only the stimulus artifact [asterisk] and no MEPs). 
$(\chi=1.457, \mathrm{p}=0.227)$, lesion site $(\chi=0.337, \mathrm{p}=0.845)$, histology $(\chi=0.281, \mathrm{p}=0.596)$, and recurrent surgery $(\chi=$ $0.067, \mathrm{p}=0.796)$.

Both LFBS and HFMS were applied in every patient; thus, it was possible to study the co-occurrence of the stimulation outcome in the same patient. The co-occurrence of task interference (Fig. 4) after LFBS and HFMS was more likely in counting $(\chi=9.36, \mathrm{p}=0.025)$ than in naming $(\chi=2.16, \mathrm{p}=0.54)$.

The average current intensity delivered by the HFMS $(2.34 \pm 0.16 \mathrm{~mA})$ was significantly higher $(\mathrm{F}(1.486)=$ $180.107, \mathrm{p}<0.001)$ than that delivered by LFBS $(1.44 \pm$ $0.14 \mathrm{~mA})$.

Motor evoked potentials were continuously monitored by stimulating the primary motor cortex with a strip electrode, as previously described. ${ }^{7}$ The MEP threshold (range 5-35 mA) was log-transformed and used as a modulation variable for the differences in the efficacy of the 2 types of stimulation. Error rates between the stimulation modalities did not vary across patients regardless of the different MEP threshold recorded, both for counting $(\chi=3.438, \mathrm{p}=$ $0.064)$ and for naming $(\chi=0.335, \mathrm{p}=0.563)$.

Intraoperative ADs and convulsive seizures were observed cumulatively in $2(4 \%)$ and $3(6 \%)$ patients, respectively, only on cortical LFBS.

The mean EOR was $95.3 \pm 15.6 \%$.

\section{Stimulation-Level Analysis}

During surgery, 1603 responses were collected. The effect of every stimulation was considered in the following ways: 1) the appropriateness of the patient's response (that

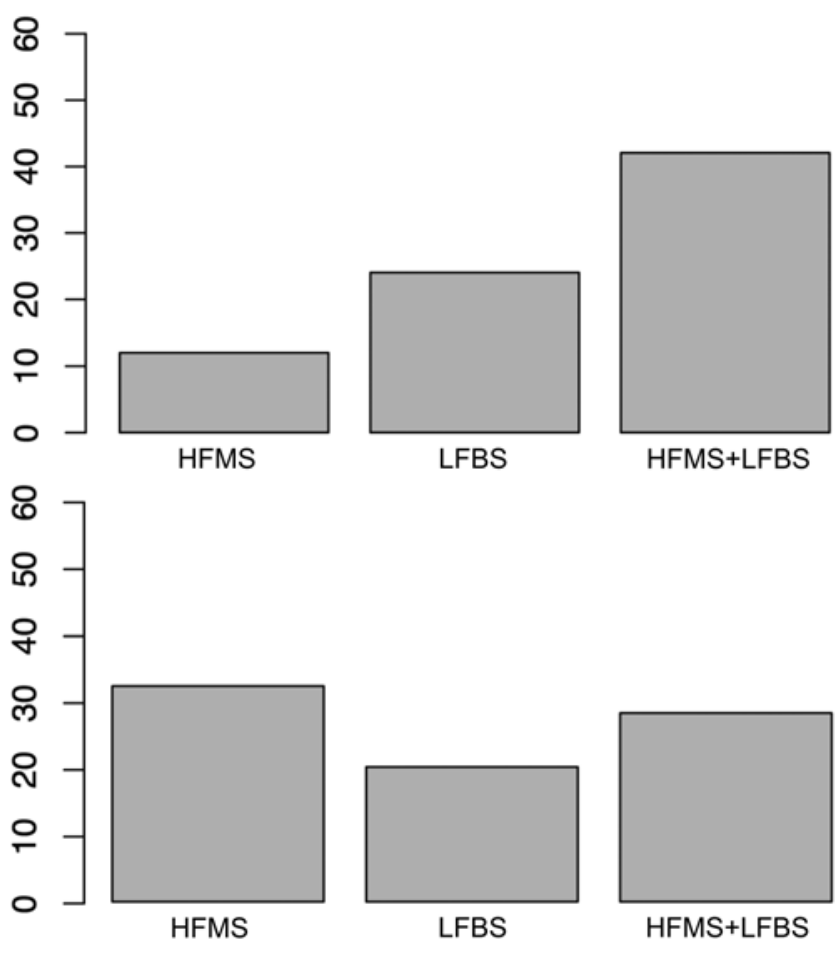

FIG. 4. Upper: Co-occurrence of stimulation-induced errors during the counting task. Lower: Co-occurrence of stimulation-induced errors during the naming task. The y-axis represents the percentage of patients.
TABLE 2. Percentage of responses by type of stimulation*

\begin{tabular}{crcc}
\hline & \multicolumn{3}{c}{ Type of Stimulation } \\
\cline { 2 - 4 } Response & Off & LFBS & HFMS \\
\hline Correct & 94 & 54 & 63 \\
\hline Incorrect & 6 & 46 & 37 \\
\hline
\end{tabular}

Correct = no language disturbance; Incorrect = any among articulatory distur-

bances, anomias, or paraphasias.

* Results are expressed as percentages.

is, correct or incorrect upon stimulation), and 2) the different outcomes determined by the 2 types of stimulation (that is, percentage of evoked errors and the type of evoked error).

Both stimulation modalities yielded a clearly different error rate as compared with baseline, when no stimulation was applied $(\chi=304.119, \mathrm{p}<0.001$; Table 2$)$. The 2 stimulation modalities slightly differed without reaching a statistically significant threshold $(\mathrm{z}=0.36, \mathrm{p}=0.06)$.

The LFBS and HFMS were then compared in terms of sensitivity and precision in producing a given language disturbance. Low-frequency bipolar stimulation showed a sensitivity of 0.458 , which was slightly higher than the HFMS sensitivity of 0.367 . Moreover, LFBS was slightly more precise $(0.665)$ than HFMS (0.582). A specificity analysis comparing the 2 stimulation modalities was not feasible because LFBS and HFMS shared the same baseline condition, that is, the absence of stimulation (stimulus off).

Evoked language disturbances were also investigated depending on their specific nature. This analysis was aimed at establishing whether the 2 stimulation modalities determined either a different percentage of errors or a different type of error. The distribution of errors was different for both LFBS $(\chi=285.405, \mathrm{p}<0.001)$ and HFMS $(\chi=$ $285.405, \mathrm{p}<0.001)$, as compared with the baseline condition of no stimulus. However, the error rate across the 3 types of language errors considered did not differ significantly between the 2 stimulation methods $(\chi=3.845, \mathrm{p}=$ 0.279; Table 3). The effect of LFBS and HFMS according to the site of stimulation is reported in Fig. 5.

\section{Is There a Target Cohort of Patients for the Use of HFMS?}

Data reported above showed HFMS to be 1) as effective as LFBS for language mapping when used at a repetition rate of $3 \mathrm{~Hz}$ and 2) less ictogenic than LFMS, although a statistical analysis could not be performed because of the limited number of recorded seizure activities.

A subset of 20 patients in Group 2 displayed distin-

TABLE 3. Type of language disturbance by stimulation modality*

\begin{tabular}{lrcc}
\hline & \multicolumn{3}{c}{ Type of Stimulation } \\
\cline { 2 - 4 } Response & Off & LFBS & HFMS \\
\hline Correct & 93.1 & 54.2 & 63.4 \\
\hline Articulatory & 0.5 & 18.5 & 15.5 \\
\hline Anomia & 2.4 & 19.6 & 11.6 \\
\hline Paraphasia & 3.3 & 7.7 & 9.7 \\
\hline
\end{tabular}

* Results are expressed as percentages. 

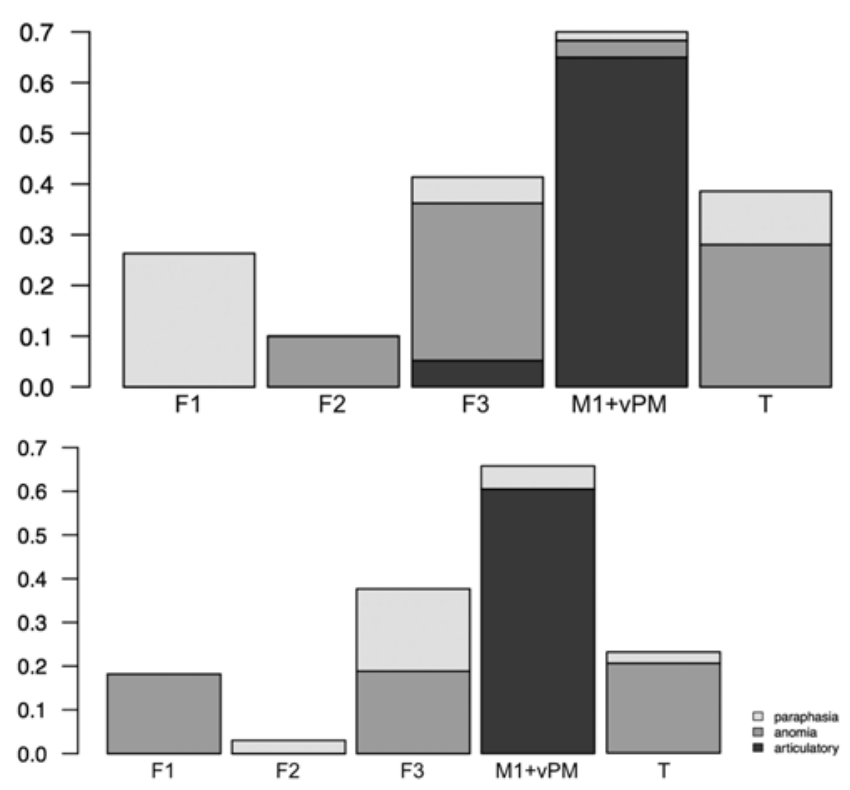

FIG. 5. Upper: Percentage of errors induced by LFBS per cortical site. Lower: Percentage of errors induced by HFMS per cortical site. A proper model aimed at testing whether the difference in error rates between the stimulation modalities varied across areas was not estimable because the cross-tabulation of cortical area, stimulations, and errors produces a large number of cells. Nonetheless, the effect of both LFBS and HFMS is described qualitatively. Performance after application of the 2 stimulation modalities did not differ greatly according to the different cortical sites explored, neither in terms of frequency or error type. F1, F2, F3 = superior, middle, and inferior frontal gyrus; M1 = primary motor area, that is, the precentral gyrus; vPM = ventral premotor area; $T$ $=$ temporal lobe.

guishing clinical features: 17 patients $(85 \%)$ had a seizure history longer than 6 months before surgery and 19 patients $(90 \%)$ were taking 2 or 3 antiepilepsy drugs. Thus, overall seizure control was difficult or poor in the majority of these patients. For this reason, they were also considered separately since this clinical profile was recently acknowledged as high risk in a large series of brain tumor patients undergoing mapping for motor function.?

When applied to the ventral premotor cortex, LFBS (median intensity $5 \mathrm{~mA}$, range $2-12 \mathrm{~mA}$ ) produced anarthria during the counting task in 11 patients (55\%) and no effect in the remaining 9 (45\%). This high rate of failed mapping with LFBS made a comparative analysis with HFMS unfeasible.

High-frequency monopolar stimulation (median intensity $10 \mathrm{~mA}$, range 4-22 $\mathrm{mA}$ ) created speech arrest in all patients, without inducing ADs. At the same current intensity, HFMS was then exclusively used to perform cortical mapping during the naming task, as described above. Functional cortical sites were identified in all patients, in either the frontal or the temporal lobes, without the occurrence of ADs or seizures. Similarly, HFMS was used for the subcortical mapping inducing anomia or phonemic or semantic paraphasias, allowing identification of the functional subcortical tracts and thus defining the resection margins in all patients.

As regards histological type, LFBS (range 2-5 mA) worked in only 4 of 12 patients affected by an HGG; in the remaining 8 patients, LFBS did not induce speech arrest even at a higher current intensity $(6-12 \mathrm{~mA})$. In the 8 patients affected by an LGG, LFBS induced speech arrest in 7 patients (2-7.5 mA) and no effect in only 1 patient (4 $\mathrm{mA})$, followed by ADs.

\section{Neuropsychological Analysis of Short- and Long-Term Outcomes}

A detailed evaluation of the different language tasks is featured in Table 4. An immediate significant postoperative (T1) decrease in test performance was observed throughout all language functions explored. At the second follow-up evaluation (T2), recovery to a level comparable with preoperative levels was recorded for all tests. A slower recovery was observed in auditory comprehension and verbal fluency tests, in which the average responses at the 1-month follow-up evaluation remained significantly lower than the baseline performance, although progressive improvement since the first postoperative evaluation was noted. Nevertheless, neuropsychological performance was above the normal scores in all tests except verbal fluency with a phonetic cue at the 1-month follow-up evaluation.

\section{Discussion}

Language is a complex function schematically composed of articulatory, phonemic, semantic, and syntactic components. ${ }^{2,5,11,12,20,24,27,28,34}$ Intraoperative testing is commonly performed with the use of DES. Low-frequency bipolar stimulation is the current gold standard technique for language mapping. ${ }^{39}$ This method of stimulation is robust and affords a high percentage of successful mappings in most patients, at both the cortical and subcortical levels. ${ }^{6,8,10,11,18,19,25,34,36}$ Afterdischarges can occasionally appear during stimulation, and they can evolve into electrical (that is, exclusively detected by ECoG) or clinical seizures. ${ }^{7}$ The incidence of seizures during awake mapping is usually quite variable, ranging from $2 \%$ to $67 \%$ based on the methodology used to detect them.,10,12,40 In the current series the seizure incidence was $11.9 \%$ when both convulsive seizures and epileptiform activity detected by ECoG were jointly considered. In patients affected by gliomas with a long history of seizures and poor seizure control, LFBS was associated with a higher risk of seizures in response to stimulation, even when the lowest useful current intensity was used. ${ }^{7}$ High-frequency monopolar stimulation has proved to be more effective than conventional LFBS in the mapping of motor structures. ${ }^{7,23,35,40}$ At present, however, very few data are available on the use of HFMS for language mapping. Previous works employing transcranial magnetic stimulation and grid electrodes ${ }^{3,32}$ have described the use of HF stimulation over the Broca's area producing speech arrest rendered results similar to those observed with LF stimulation. Our study confirmed these observations intraoperatively.

The ability of HFMS at $3 \mathrm{~Hz}$ to efficiently map the components of language was explored in a prospective cohort mainly affected by dominant frontal and temporal tumors. These patients, given their clinical, radiological, and neuropsychological features, were the best candidates for conventional LFBS. Therefore, a comparison 
TABLE 4. Analysis of the neuropsychological outcomes*

\begin{tabular}{|c|c|c|c|c|c|c|c|c|c|c|}
\hline \multirow[b]{2}{*}{ Test Type } & \multirow{2}{*}{$\begin{array}{l}\text { Normal } \\
\text { Value }\end{array}$} & \multirow[b]{2}{*}{ TO† } & \multirow[b]{2}{*}{ T1† } & \multirow[b]{2}{*}{$\mathrm{T} 2 \dagger$} & \multicolumn{2}{|c|}{ T0 vs T1 } & \multicolumn{2}{|c|}{ T0 vs T2 } & \multicolumn{2}{|c|}{ ANOVA } \\
\hline & & & & & p Value & t Value & p Value & t Value & $p$ Value & F Value \\
\hline Auditory comprehension & $>26.5$ & 31.2 & 20.6 & 27.6 & $<0.001$ & $(68,9)=-9.7$ & 0.008 & $(73,4)=-2.7$ & $<0.001$ & $(2,71)=47.7$ \\
\hline Object naming & $87.3 \%$ & 93.1 & 72.9 & 88.1 & $<0.001$ & $(68,9)=-5.2$ & 0.126 & $(72,3)=-1.6$ & $<0.001$ & $(2,70)=13.8$ \\
\hline Action naming & $73.4 \%$ & 87.9 & 68.9 & 84.5 & $<0.0001$ & $(66,7)=-5.1$ & 0.319 & $(70,2)=-1.0$ & $<0.001$ & $(2,69)=13.8$ \\
\hline \multicolumn{11}{|l|}{ Verbal fluency } \\
\hline Semantic cue & $>24$ & 37.3 & 17.7 & 26.8 & $<0.001$ & $(67,1)=-9.7$ & $<0.001$ & $(70,8)=-4.4$ & $<0.001$ & $(2,69)=46.8$ \\
\hline Phonetic cue & $>16$ & 27.4 & 9.7 & 15.5 & $<0.001$ & $(70,4)=-9.7$ & $<0.001$ & $(72,6)=-5.5$ & $<0.001$ & $(2,71)=47.9$ \\
\hline Word comprehension & $>96 \%$ & 99.7 & 93.8 & 96.9 & 0.027 & $(67,5)=-2.3$ & 0.307 & $(74,2)=-1.0$ & 0.085 & $(2,72)=2.6$ \\
\hline Sentence comprehension & $>90 \%$ & 96.8 & 82.3 & 93.7 & $<0.001$ & $(53,9)=-4.0$ & 0.651 & $(56,5)=-0.5$ & $<0.001$ & $(2,58)=9.8$ \\
\hline
\end{tabular}

$\mathrm{T} 0=$ preoperative evaluation; $\mathrm{T} 1$ = evaluation at 1 week since surgery; $\mathrm{T} 2$ = evaluation at 3 months since surgery.

* Bold values are statistically significant and values within parentheses represent degrees of freedom.

† Mean values.

between the 2 techniques was expected to lead to reliable findings, although patient selection biases cannot be completely ruled out. No difference in the rate, site, and stability of response was observed when the 2 techniques were compared within each patient. Current data showed that HFMS, compared with motor mapping, can robustly generate a wide spectrum of language interference at a higher repetition rate (that is, $3 \mathrm{~Hz}$ ), which is needed to interfere with the more associative component of language, such as semantics and phonetics. The current intensity needed to generate language disturbances with HFMS is usually higher than that needed with LFBS, demonstrating a linear relationship $\left(r^{2}=0.515\right.$; Fig. 6$)$; on average, double the intensity of that required for LFBS is required to obtain comparable effectiveness with HFMS.

Mapping during the resection of tumors affecting language areas and pathways has multiple aims: 1) to identify eloquent cortical sites and a safe entry point, and 2) to find the essential subcortical structures defining the functional boundaries and EOR. Resection performed according to the functional boundaries implies reaching essential subcortical tracts, characterized by the sudden appearance of various language disturbances, each one typically associated with each tract. ${ }^{6,7,18,19}$ Functional resection in the immediate postoperative period is associated with the occurrence of language deficits, generally starting in the 1st or 2 nd postoperative days and progressively recovering within 1 or 2 weeks from surgery. In fact, the rate of permanent deficits is usually $2 \%$ in most series, $6,7,10,13,17-19,31,34,36$ and most patients have a complete recovery 3 months after surgery. The thorough neuropsychological evaluation performed in our series showed that HFMS can identify the essential subcortical tracts as efficiently as LFBS, determining a high percentage of complete resection and an optimal postoperative functional outcome, as supported by data regarding the EOR and the distinct language domains tested at the 3 time points. Such an extensive neuropsychological evaluation significantly highlighted how distinct components of language behave differently following surgery, especially in the postoperative recovery. In particular, object and action naming and sentence comprehension reached a similar level of proficiency compared with baseline after 1 month postsurgery, whereas fluency and auditory processing remained impaired at a midterm follow-up evaluation, although they were slowly recovering compared with the immediate postoperative levels. These results also highlighted the relevance of a wide analysis of language performance in patients undergoing this type of surgery to detect as many treatment effects as possible. The current data showed that while LFBS remains the neurophysiological standard, HFMS can be regarded as an efficient and safe alternative in patients considered at high risk because of their clinical and radiological characteristics, in whom, for instance, a long history of seizures and poor seizure control are present. In these patients, LFBS can be associated with a higher risk of seizures in response to stimulation, even when stimulation at the lowest working intensity is used.7,40 The epileptic threshold for LFBS may be very close to or even lower than that inducing a language response; therefore, the chance of performing effective functional mapping may be limited with a high rate of failure, as found in the current study. In patients with such a profile, LFBS mapping could not be completed and yielded false-negative results when applied to the ventral premotor cortex in up to $45 \%$ cases. High-frequency monopolar stimulation was then used as the exclusive tech-

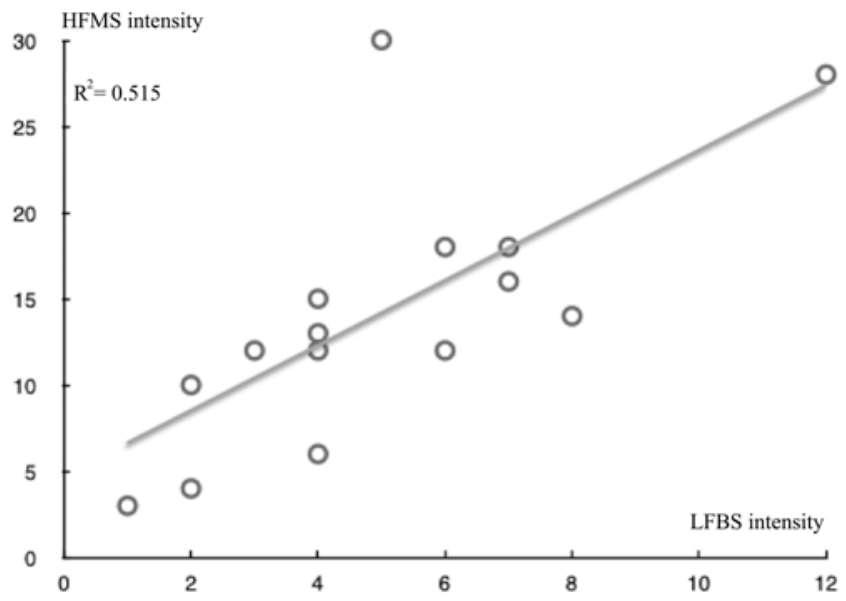

FIG. 6. Relationship between the current intensity $(\mathrm{mA})$ of LFBS and HFMS. 
nique of DES, enabling successful mapping of the different language components.

The lower ictogenicity of HFMS may be mainly attributable to the fact that it delivers very short trains (10.5-18.5 $\mathrm{msec})$ of high-frequency $(250-500 \mathrm{~Hz})$ stimuli. Instead, long trains (1-4 seconds) of $60-\mathrm{Hz}$ stimuli characterize LFBS. Although the current intensity is significantly higher when using HFMS, it is applied for a much shorter time than LFBS and thus delivers less electric charge to the tissue. The HFMS may also be less ictogenic because the stimulation is less concentrated over tissue with aberrant excitability.

Some limitations of this study must be acknowledged. In fact, a more detailed neurophysiological understanding of the stimulation effects on the axons of different neurons is still lacking, and thus a different type of analysis is required to better elucidate this relationship. ${ }^{21} \mathrm{~A}$ blinded comparison between the 2 stimulation modalities should be performed to address this issue in a more robust way. However, such a study is unlikely to be easily realized with appropriate statistical power for the following reasons. First, LFBS remains the gold-standard DES technique, ${ }^{12}$ and as such, a patient cannot be deprived of this technique. Second, large multicenter studies are required to perform such an extensive blinded trial given the epidemiology of intrinsic brain tumors. Moreover, HFMS is less available throughout distinct centers given its status as a relatively newer technique than LFBS. Spatial accuracy may also be limited with HFMS because of possible current spreading, and thus affecting a greater cortical area. Finally, more extensive experience with this type of stimulation is advocated to refine these data.

\section{Conclusions}

This work shows that language mapping performed with HFMS at a repetition rate of $3 \mathrm{~Hz}$ is feasible and effective, both at the cortical and subcortical levels, with a level of proficiency comparable to that for LFBS. Given its lower ictogenicity, HFMS can be successfully applied to patients with a long history of seizures and poor seizure control. In such patients, LFBS can be associated with a higher incidence of epileptic events negatively affecting the success rate of complete language mapping and eventually the EOR. High-frequency monopolar stimulation can be regarded as an additional tool for the resection of brain lesions in eloquent areas during awake anesthesia. It can thus help to achieve a complete EOR and full preservation of a patient's functional integrity. It can also represent a neurophysiological tool for investigating the neural bases of language.

\section{Acknowledgments}

The patients and their families are warmly acknowledged for their cooperation and support. We would also like to extend our thanks to all staff of the operating room and the ward for their invaluable efforts in the care and management of the patients and for their support of the surgical and research activities.

Dr. Riva was supported by Fellowship for Abroad 2013 of the Fondazione Italiana per la Ricerca sul Cancro (FIRC). This work was supported by grants from Ricerca Finalizzata, Ministero della Salute 2009 and 2010, and Fondazione Berlucchi (L.B.).

\section{References}

1. Allison PD: Event History Analysis: Regression for Longitudinal Event Data. Newbury Park, CA: Sage Publications, 1984

2. Amunts K, Zilles K: Architecture and organizational principles of Broca's region. Trends Cogn Sci 16:418-426, 2012

3. Axelson HW, Hesselager G, Flink R: Successful localization of the Broca area with short-train pulses instead of 'Penfield' stimulation. Seizure 18:374-375, 2009

4. Bello L, Castellano A, Fava E, Casaceli G, Riva M, Scotti $\mathrm{G}$, et al: Intraoperative use of diffusion tensor imaging fiber tractography and subcortical mapping for resection of gliomas: technical considerations. Neurosurg Focus 28(2):E6, 2010

5. Bello L, Gallucci M, Fava M, Carrabba G, Giussani C, Acerbi F, et al: Intraoperative subcortical language tract mapping guides surgical removal of gliomas involving speech areas. Neurosurgery 60:67-82, 2007

6. Bello L, Gambini A, Castellano A, Carrabba G, Acerbi F, Fava E, et al: Motor and language DTI Fiber Tracking combined with intraoperative subcortical mapping for surgical removal of gliomas. Neuroimage 39:369-382, 2008

7. Bello L, Riva M, Fava E, Ferpozzi V, Castellano A, Raneri F, et al: Tailoring neurophysiological strategies with clinical context enhances resection and safety and expands indications in gliomas involving motor pathways. Neuro Oncol 16:1110-1128, 2014

8. Berger MS, Ojemann GA: Intraoperative brain mapping techniques in neuro-oncology. Stereotact Funct Neurosurg 58:153-161, 1992

9. Capelle L, Fontaine D, Mandonnet E, Taillandier L, Golmard JL, Bauchet L, et al: Spontaneous and therapeutic prognostic factors in adult hemispheric World Health Organization Grade II gliomas: a series of 1097 cases: clinical article. J Neurosurg 118:1157-1168, 2013

10. Chang EF, Clark A, Smith JS, Polley MY, Chang SM, Barbaro NM, et al: Functional mapping-guided resection of low-grade gliomas in eloquent areas of the brain: improvement of longterm survival. Clinical article. J Neurosurg 114:566-573, 2011

11. Chang EF, Wang DD, Perry DW, Barbaro NM, Berger MS: Homotopic organization of essential language sites in right and bilateral cerebral hemispheric dominance. J Neurosurg 114:893-902, 2011

12. Deras P, Moulinié G, Maldonado IL, Moritz-Gasser S, Duffau $\mathrm{H}$, Bertram L: Intermittent general anesthesia with controlled ventilation for asleep-awake-asleep brain surgery: a prospective series of 140 gliomas in eloquent areas. Neurosurgery 71:764-771, 2012

13. De Witt Hamer PC, Robles SG, Zwinderman AH, Duffau H, Berger MS: Impact of intraoperative stimulation brain mapping on glioma surgery outcome: a meta-analysis. J Clin Oncol 30:2559-2565, 2012

14. Dobson AJ, Barnett AG: An Introduction to Generalized Linear Models, ed 3. Boca Raton, FL: Chapman \& Hall/ CRC, 2008

15. Duffau H: Lessons from brain mapping in surgery for lowgrade glioma: insights into associations between tumour and brain plasticity. Lancet Neurol 4:476-486, 2005

16. Duffau H, Capelle L: Preferential brain locations of low-grade gliomas. Cancer 100:2622-2626, 2004

17. Duffau H, Capelle L, Denvil D, Sichez N, Gatignol P, Lopes $\mathrm{M}$, et al: Functional recovery after surgical resection of low grade gliomas in eloquent brain: hypothesis of brain compensation. J Neurol Neurosurg Psychiatry 74:901-907, 2003

18. Duffau H, Capelle L, Denvil D, Sichez N, Gatignol P, Taillandier L, et al: Usefulness of intraoperative electrical subcortical mapping during surgery for low-grade gliomas located within eloquent brain regions: functional results in a 
consecutive series of 103 patients. J Neurosurg 98:764-778, 2003

19. Duffau H, Capelle L, Sichez N, Denvil D, Lopes M, Sichez $\mathrm{JP}$, et al: Intraoperative mapping of the subcortical language pathways using direct stimulations. An anatomo-functional study. Brain 125:199-214, 2002

20. Graziano MS, Aflalo TN: Mapping behavioral repertoire onto the cortex. Neuron 56:239-251, 2007

21. Histed MH, Ni AM, Maunsell JH: Insights into cortical mechanisms of behavior from microstimulation experiments. Prog Neurobiol 103:115-130, 2013

22. Kleihues P, Louis DN, Scheithauer BW, Rorke LB, Reifenberger G, Burger PC, et al: The WHO classification of tumors of the nervous system. J Neuropathol Exp Neurol 61:215229, 2002

23. Kombos T, Suess O, Kern BC, Funk T, Hoell T, Kopetsch O, et al: Comparison between monopolar and bipolar electrical stimulation of the motor cortex. Acta Neurochir 141:12951301, 199

24. Matsumoto R, Nair DR, LaPresto E, Najm I, Bingaman W, Shibasaki H, et al: Functional connectivity in the human language system: a cortico-cortical evoked potential study. Brain 127:2316-2330, 2004

25. Ojemann G, Ojemann J, Lettich E, Berger M: Cortical language localization in left, dominant hemisphere. An electrical stimulation mapping investigation in 117 patients. J Neurosurg 71:316-326, 1989

26. Pallud J, Audureau E, Blonski M, Sanai N, Bauchet L, Fontaine D, et al: Epileptic seizures in diffuse low-grade gliomas in adults. Brain 137:449-462, 2014

27. Papagno C, Casarotti A, Comi A, Gallucci M, Riva M, Bello L: Measuring clinical outcomes in neuro-oncology. A battery to evaluate low-grade gliomas (LGG). J Neurooncol 108:269-275, 2012

28. Papagno C, Gallucci M, Casarotti A, Castellano A, Falini A, Fava E, et al: Connectivity constraints on cortical reorganization of neural circuits involved in object naming. Neuroimage 55:1306-1313, 2011

29. Papagno C, Miracapillo C, Casarotti A, Romero Lauro LJ, Castellano A, Falini A, et al: What is the role of the uncinate fasciculus? Surgical removal and proper name retrieval. Brain 134:405-414, 2011

30. Quiñones-Hinojosa A, Ojemann SG, Sanai N, Dillon WP, Berger MS: Preoperative correlation of intraoperative cortical mapping with magnetic resonance imaging landmarks to predict localization of the Broca area. J Neurosurg 99:311-318, 2003

31. Riva M, Bello L: Low-grade glioma management: a contemporary surgical approach. Curr Opin Oncol 26:615-621, 2014

32. Rogić M, Deletis V, Fernández-Conejero I: Inducing transient language disruptions by mapping of Broca's area with modified patterned repetitive transcranial magnetic stimulation protocol. J Neurosurg 120:1033-1041, 2014

33. Sanai N, Berger MS: Glioma extent of resection and its impact on patient outcome. Neurosurgery 62:753-764, 264266,2008
34. Sanai N, Mirzadeh Z, Berger MS: Functional outcome after language mapping for glioma resection. N Engl J Med 358:18-27, 2008

35. Seidel K, Beck J, Stieglitz L, Schucht P, Raabe A: Lowthreshold monopolar motor mapping for resection of primary motor cortex tumors. Neurosurgery 71 (1 Suppl Operative): $104-115,2012$

36. Smith JS, Chang EF, Lamborn KR, Chang SM, Prados MD, Cha S, et al: Role of extent of resection in the long-term outcome of low-grade hemispheric gliomas. J Clin Oncol 26:1338-1345, 2008

37. Soffietti R, Baumert BG, Bello L, von Deimling A, Duffau $\mathrm{H}$, Frénay M, et al: Guidelines on management of low-grade gliomas: report of an EFNS-EANO Task Force. Eur J Neurol 17:1124-1133, 2010

38. Stupp R, Mason WP, van den Bent MJ, Weller M, Fisher B, Taphoorn MJ, et al: Radiotherapy plus concomitant and adjuvant temozolomide for glioblastoma. N Engl J Med 352:987-996, 2005

39. Szelényi A, Bello L, Duffau H, Fava E, Feigl GC, Galanda M, et al: Intraoperative electrical stimulation in awake craniotomy: methodological aspects of current practice. Neurosurg Focus 28(2):E7, 2010

40. Szelényi A, Senft C, Jardan M, Forster MT, Franz K, Seifert $\mathrm{V}$, et al: Intra-operative subcortical electrical stimulation: a comparison of two methods. Clin Neurophysiol 122:14701475,2011

41. Taniguchi M, Cedzich C, Schramm J: Modification of cortical stimulation for motor evoked potentials under general anesthesia: technical description. Neurosurgery 32:219-226, 1993

42. Tate MC, Guo L, McEvoy J, Chang EF: Safety and efficacy of motor mapping utilizing short pulse train direct cortical stimulation. Stereotact Funct Neurosurg 91:379-385, 2013

\section{Disclosure}

The authors report no conflict of interest concerning the materials or methods used in this study or the findings specified in this paper.

\section{Author Contributions}

Conception and design: Bello, Riva. Acquisition of data: Bello, Riva, Fava, Comi, Casarotti, Alfiero, Raneri, Pessina. Analysis and interpretation of data: Bello, Riva, Fava, Gallucci, Comi. Drafting the article: Bello, Riva, Fava. Critically revising the article: Bello, Riva, Fava, Pessina. Reviewed submitted version of manuscript: Riva, Gallucci, Comi, Raneri. Approved the final version of the manuscript on behalf of all authors: Bello. Statistical analysis: Riva, Gallucci. Study supervision: Bello.

\section{Correspondence}

Lorenzo Bello, Surgical Neuro-Oncology, Università degli Studi di Milano, Humanitas Research Hospital, Via Manzoni 56, Rozzano 20142, Italy. email: lorenzo.bello@unimi.it. 